www.jmscr.igmpublication.org

Impact Factor 5.244

Index Copernicus Value: 83.27

ISSN (e)-2347-176x ISSN (p) 2455-0450

crossref DOI:_http://dx.doi.org/10.18535/jmscr/v4i7.78

Journal Of Medical Science And Clinical Research

IGM Publication

An Official Publication of IGM Publication

\title{
Efficiency of Bilateral Subcostal Transversus Abdominis Plane Block to Complement General Anaesthesia in Laparoscopic Cholecystectomy
}

Authors

\author{
Davies C. Vergheese ${ }^{1}$, Kamal Sonya ${ }^{2}$, Reshmi Suprakash ${ }^{3}$ \\ ${ }^{1}$ Associate professor, ${ }^{2}$ Professor, ${ }^{3}$ Senior Resident \\ Jubilee Mission Medical College and Research Institute, Thrissur, Kerala, India \\ Corresponding Author \\ Davies C. Vergheese \\ Email:daviescv@gmail.com
}

\begin{abstract}
Introduction: Laparoscopic Cholecystectomy is presently a commonly performed surgical procedure. It is needed in both the young and the old; healthy as well as the severely co-morbid. During the surgery, pain is generated at the port site and also from the viscera. Bilateral Subcostal (Upper Quadrant) Transversus Abdominis Plain Block can block the pain from the parietal port sites. This study is to assess the efficiency of Bilateral Subcostal Transversus Abdominis Plane Block as a mode of multi-modal analgesia in the General Anaesthesia administered for Laparoscopic Cholecystectomy.

Material and Methods: One hundred patients of ASA 1 or 2 categories in the age group 20 to 60 years from both sex posted for elective Laparoscopic Cholecystectomy was included in the study. Fifty patients each was randomly assigned to two groups ' $A$ ' and ' $B$ '. Group A was the control group and did not receive any blocks. The surgery was done solely under General Anaesthesia. In the group 'B', Bilateral Subcostal TAP block with $30 \mathrm{ml}$ of $0.25 \%$ Ropivacaine was administered from the Block Room. General Anaesthesia in a standardised protocol for the study was administered by a different Anaesthetist who was blinded about the blocks. The extra amount of Fentanyl required per hour to maintain adequate anaesthesia was noted in every patient. Minimum concentration of Sevoflurane demanded by individual patients is also noted. Systolic BP value range of 'Control BP to an additional $15 \mathrm{~mm}$ of $\mathrm{Hg}$ ' is targeted as adequate anaesthetic depth in this study.

Post Operative Analgesia also had a standard protocol for all the patients. The additional dose Inj. Tramadol to control the pain in the 8 post operative hours is also noted.

Results: The study demonstrated a statistically significant decrease in the intra operative inhalational anaesthetic and narcotic analgesic demand, in the Group B in which Bilateral Subcostal TAP Block was administered. The decrease in the 8 hour post operative analgesic demand in the Group B was found to be statistically significant.
\end{abstract}

Conclusion: After a bilateral Subcostal TAP block, there is a decrease in the intra operative anaesthetic demand for a Laparoscopic Cholecystectomy. The regional analgesia extends to immediate 8 post operative hours, and reduces the analgesic demand. This may not make a marked difference in the outcome in ASA 1 or 2 patients. But in ASA 3 or 4 patients with poor cardiac or other system reserve, a higher narcotic analgesic or inhalational anaesthetic requirement may cause decompensation. A low anaesthetic demand after a Bilateral Subcostal TAP block may be beneficial in these patients.

Keywords-Laparoscopic Cholecystectomy, Subcostal TAP Block, Multimodal Analgesia, Sevoflurane, Ropivacaine, Fentanyl, Tramadol. 


\section{INTRODUCTION}

Laparoscopic Cholecystectomy is a commonly performed surgical procedure. It is done not only in the healthy or young individuals but in the severely co-morbid and elderly also. Pneumoperitoneum induced for the procedure add on to the anaesthetic risk. The high pressure in the abdominal cavity retards the venous return and increases the afterload to the heart. Higher intrathoracic pressure is required for adequate ventilation. In addition, higher minute ventilation is needed to wash out the Carbon Dioxide absorbed from the peritoneal cavity. Head up position required for the procedure further reduces the venous return and cardiac output. This additional cardiopulmonary disturbance imposed by the pneumoperitoneum is well tolerated by the healthy adults. But this may be detrimental in those with poor cardio-pulmonary and respiratory function.

Inhalational General Anaesthetics have a negative ionotropic effect, and also reduces the cardiac preload and afterload which may lead to cardiovascular failure in those with poor cardiac function. Reduction of the Inhalational Anaesthetic concentration may be desirable in these patients.

Many a time patients with Cardiac Regional Wall Movement Abnormalities with an Ejection Fraction less than 30\% need Laparoscopic Cholecystectomy. Transversus Abdominis Plane Block is a compartment block where the Local Anaesthetic in large volume is injected into the tissue plane between the Internal Oblique and Transversus Abdominis muscles where the neurovascular bundles to the abdominal wall ply. TAP block provides sensory and motor blockade to the structures including and external to the parietal peritoneum. The classical TAP Block administered above the level of the iliac crest provides analgesia in the segments T10 to L 1 levels. Classical TAP can be administered by landmark based double pop Loss Of Resistance technique. Ultrasound guidance gives a precision block. It does not provide analgesia above the umbilicus. The spread of dye injected in cadaver studies correlates with this observation $^{2}$. The beneficial effects of the
'Classical' or the Lower Quadrant TAP block as a mode of post operative analgesia for infraumbilical laprotomies, Appendicectomy, and Caesarean Section have been demonstrated by many researchers ${ }^{4,5,6}$.

The Ultrasound guided Subcostal TAP introduced in 2008 provide analgesia above the umbilicus.. A higher volume in this location will spread to levels from $\mathrm{T} 6$ to $\mathrm{L}^{7}$.

Bilateral Subcostal TAP block is likely to prevent pain from the port sites not only at the time of introduction but also all through the surgery during the manipulation of the instruments. The major portion of the pain during the laparoscopic procedure is generated from the abdominal wall. The visceral pain is not caused by cutting, but only by squeezing and pulling which can be easily controlled by a smaller dose of inhalational agent and narcotic analgesic. The motor blockade provided by the TAP Block may lower intra abdominal pressures required for adequate distension. There are many studies substantiating the effectiveness of Subcostal TAP for Laparoscopic Cholecystectomy ${ }^{7,8,10,11}$. Systemic review on TAP block ${ }^{3}$ published in 2012 shows that a pre procedural TAP block provides more effective post operative analgesia, possibly because of the pre emptive effects. Most of the studies evaluated the post operative analgesia only. Right unilateral Subcostal Tap Block as done in one study ${ }^{8}$. It may provide inadequate analgesia in the midline ports.

In another study ${ }^{10,12}$, a single injection Subcostal TAP block was found more effective than IV opioid analgesia, while continuous thoracic epidural analgesia was found more effective than the single injection subcostal TAP block. But, a continuous thoracic epidural may be too invasive for a minimally invasive procedure which causes a relatively mild pain than an open surgery.

Port site infiltration ${ }^{8}$ and intraperitoneal instllation ${ }^{9}$ with Local Anaesthetics did not exhibit significant post operative analgesia.

The efficiency of Bilateral Subcostal TAP Block on the intra operative anaesthetic requirement and the first 8 hours post operative analgesic requirement 
for Laparoscopic Cholecystectomy is aimed in our study.

\section{MATERIAL AND METHODS}

Study design: Randomized prospective control double blinded study done after institutional ethical committee approval and informed written consent from the patients. One hundred patients admitted for elective Laparoscopic Cholecystectomy from February 2013 to April 2014 was included in this study. Fifty patients each was randomly assigned to two groups ' $\mathrm{A}$ ' and ' $\mathrm{B}$ '.

Group A - In 50 patients, the surgery was done solely under General Anaesthesia without a TAP Block.

Group B - In 50 patients, Sub-costal TAP Block was administered with $30 \mathrm{ml}$ of $0.25 \%$ Ropivacaine from the Block Room.

\section{Inclusion criteria-}

Age group 20 to 60 years and belonging to ASA grade 1 or 2 .

\section{Exclusion}

Laparoscopic procedure converted to open .

Pregnancy, Co-morbid conditions -CAD, CRF, COPD patients, Psychiatric illness and Neuromuscular disorder.

\section{Procedure}

Systolic BP is noted from the pre anaesthetic area, before the start of any painful interventions. It is compared with that recorded in the pre anaesthetic chart and the nursing record. If there is a marked difference, the examination is repeated, to rule out a pre operative hike due to anxiety. 'Control Systolic BP' is found out and recorded, which is the reference level to maintain adequate anaesthetic levels.

Group B patients were brought to the Block Room for the administration of the bilateral Sub-costal TAP Block under the ultrasound guidance. All standard monitoring including $\mathrm{SPO}_{2}, \mathrm{ECG}$, NonInvasive BP were connected.
Intravenous access was established. Procedural sedation with titrated doses up to a maximum of Midazolam $2 \mathrm{mg}$ and Dexmedetomidine $10 \mathrm{mcg}$ was administered.

The patient lies in the supine position on the table. Abdomen is cleaned with spirit and later with Chlorhexidine solution. The abdomen is covered with a fenestrated plastic sterile drape.

Scanning- A Sonosite M Turbo Ultrasound Machine is used for the USG Block. The linear probe $6-13 \mathrm{MHz}$ is sheathed with a sterile camera cover. The operator stands on the left side of the patient. The probe is placed over right the subcostal area near the midline with the orientation marker medially. The Rectus muscle inside the sheath is identified.

Fig 1 Left Subcostal TAP Block;

Scanning and in-plane needling.

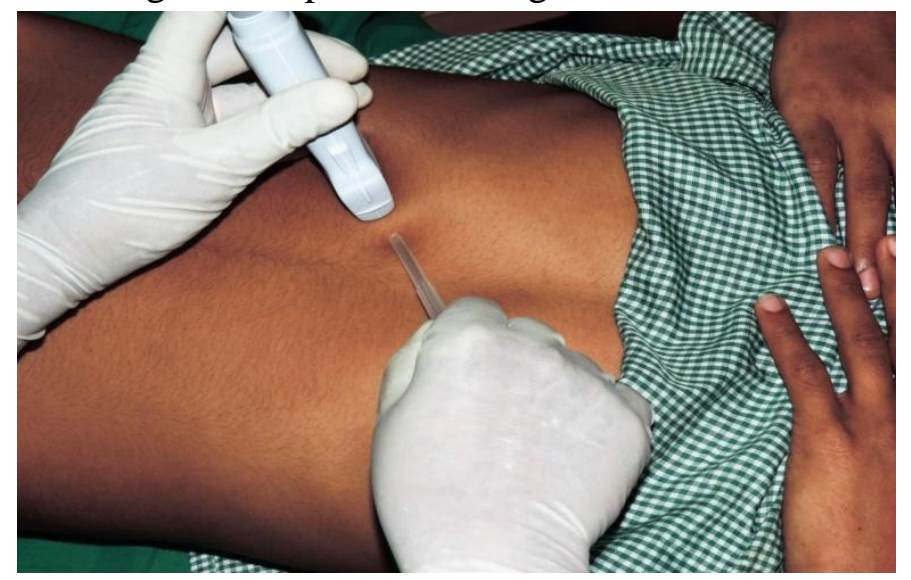

The External Oblique, Internal oblique and the Transversus Abdominis muscles are found attaching to the lateral aspect of the Rectus sheath. The Transversus Abdominis muscle is seen to extend medially under the posterior Rectus sheath. The site of injection of the local anaesthetic in the Sub-costal TAP block is under the Posterior Rectus sheath and above the Transversus Abdominis muscle. 
Fig 2 Ultrasonogram of Subcostal TAP;

In-plane needling.

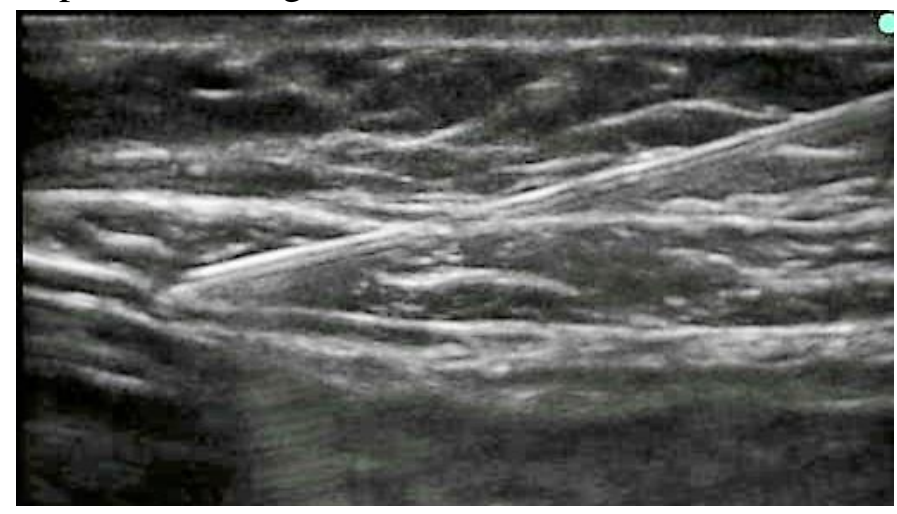

Needle tip on the Posterior Rectus Sheath.

Transversus Abdominis Muscle is seen under.

Needling- Is done in-plane from medial to inferolateral direction keeping the Ultrasound probe close to the costal margin on the right side. The needle traverses the skin, sub-cutaneous tissue, anterior rectus sheath, rectus muscle, posterior rectus sheath and reaches the TAP plane where the $30 \mathrm{ml}$ of local anaesthetic Ropivacaine $0.25 \%$ is injected. The drug separates the Transversus Abdominis muscle from the posterior Rectus sheath. The needle is advanced further infero-laterally as the plane is opened up. The procedure is repeated on the left side.

The patient is transferred to the Operating Theatre 30 minutes after the block.

The Group A patients are taken up without the block.

\section{General Anaesthesia by standardized protocol-}

In the Operation Theatre, a different Anaesthetist who is blinded about the block, administer anaesthesia, by a standardized protocol. Intravenous access with a cannula is done if not already established. Monitoring- $\mathrm{SPO}_{2}, \mathrm{ECG}$, Non invasive $\mathrm{BP}$, and Capnograph are used.

Injection Fentanyl $1 \mathrm{mcg} / \mathrm{kg}$ body weight, Inj. Glycopyrrolate $0.4 \mathrm{Mg}$ and Inj Ondansetron $8 \mathrm{Mg}$ is administered slow IV. Atracurium 0.5 Mg IV is injected. One minute after Atracurium, Inj. Propofol $1 \mathrm{Mg} / \mathrm{Kg}$ is given slow IV. Assisted and later Intermittent Positive Pressure Ventilation with a mask is started with $2 \%$ Sevoflurane in $50 \%$ Nitrous
Oxide in Oxygen. When the muscle relaxation is adequate at 3-5 minutes after Atracurium, an additional dose of Propofol, $1 \mathrm{mg} / \mathrm{kg}$ is injected. Laryngoscopy and tracheal intubation is done with a cuffed endotracheal tube of appropriate size. Intermittent positive Pressure Ventilation with 2\% Sevoflurane in $66 \%$ Nitrous Oxide in Oxygen is started. Atracurium is repeated at a dose of $1 / 4$ of the intubating dose every 20 minutes or earlier if needed. Fentanyl in a dose of $0.25 \mathrm{mcg} / \mathrm{kg}$ is repeated 30 minutes after the previous dose.

The changes in the systolic BP during anaesthesia are utilized to assess the anaesthetic depth required during different phases of surgery. In the 'Patient Response to Surgical Stimulus' (PSRT) Scoring system to assess the anaesthetic depth, systolic BP is considered as one of the important parameters ${ }^{13}$, "Control SBP +15 mmof $\mathrm{Hg}$ " is given a score of ' 0 '. Systolic BP value range of 'Control $+15 \mathrm{~mm}$ of $\mathrm{Hg}$ ', is targeted as adequate anaesthetic depth in this study.

Titration of anaesthetic depth was done by a standardized protocol so that the total consumption of Fentanyl and minimum concentration of Sevoflurane derived will indicate intra operative anaesthetic demand. Additional dose of Fentanyl or the minimum Sevoflurane concentration is exercised to bring the SBP to this targeted range is recorded. An SBP $15 \mathrm{~mm}$ of $\mathrm{Hg}$ above the control value 10 minutes after the previous Fentanyl dose attracted an additional aliquot of $0.25 \mathrm{mcg} / \mathrm{kg}$ Fentanyl. With a SBP fall by $15 \mathrm{~mm}$ of $\mathrm{Hg}$ from the control value the concentration of Sevoflurane is reduced by $0.5 \%$. If the value remains less than 15 $\mathrm{mm}$ of $\mathrm{Hg}$ of the control $10 \mathrm{~min}$ after reduction, Sevoflurane concentration is further reduced by $0.5 \%$. If the SBP rises later, the Sevoflurane Concentration is raised by $0.5 \%$ at a time upto $2 \%$; before additional aliquots of Fentanyl is administered. The minimum Sevoflurane concentration demanded by the patient and the total Fentanyl consumption are charted. Fentanyl consumption per hour per $\mathrm{kg}$ body weight is calculated by dividing the total consumption by duration in hours. A lower Fentanyl consumption 
and a lower minimum Sevoflurane demanded means lower anaesthetic requirement.

The surgeon is requested to give port site infiltration with or $0.25 \%$ Bupivacaine before the skin closure which is the standard institutional practice after all laparoscopic surgeries.

At the end of the surgery, the residual neuromuscular blockade is reversed with Glycopyrrolate and Neostigmine and trachea extubated.

\section{Post- operative Analgesia}

Inj. Tramadol $0.5 \mathrm{Mg} / \mathrm{Kg}$ slow IV in the immediate post operative period when the patient is fully recovered from anaesthesia. This is to reduce the emergence phenomenon and the rebound pain that can occur by the abrupt withdrawal of the analgesic support by the short acting Fentanyl and Sevoflurane.

An additional $0.5 \mathrm{mg} / \mathrm{kg}$ aliquots were administered IV after a lock out time of 30 minutes if the patient complains of pain. Post operative Tramadol requirement for the first 8 hours is noted as a measure of additional post operative analgesic requirement.

\section{RESULTS}

The patient data are presented below.

Table:1 Age Distribution of Patients

\begin{tabular}{|l|c|c|}
\hline Age & Group A & Group B \\
\hline $20-30$ & 8 & 7 \\
\hline $30-40$ & 17 & 24 \\
\hline $40-50$ & 14 & 10 \\
\hline $50-60$ & 12 & 9 \\
\hline TOTAL & 50 & 50 \\
\hline
\end{tabular}

Table:2 Mean and Standard Deviation of Age In Group A \& B

\begin{tabular}{|l|c|c|}
\hline Age & Group A & Group B \\
\hline Mean & 43.88 & 40.02 \\
\hline SD & 12.5 & 11.2 \\
\hline
\end{tabular}

Both groups are of similar age group.
Table:3 Sex distribution of patients of A and B

\begin{tabular}{|l|c|c|}
\hline Sex & Group A & Group B \\
\hline Male & 18 & 14 \\
\hline Female & 32 & 36 \\
\hline
\end{tabular}

There is a female preponderance in both the groups which is a known and accepted incidence pattern in cholelithiasis.

Table:4 Duration of surgery of patients of A and B in minutes.

\begin{tabular}{|l|c|c|}
\hline Duration & A & B \\
\hline Mean & 80.98 & 84.28 \\
\hline SD & 6.01 & 8.53 \\
\hline
\end{tabular}

There is no significant difference between average duration of surgery of Group A and Group B

Table:5 ASA status of patients of A and B

\begin{tabular}{|l|l|l|}
\hline ASA & A & B \\
\hline 1 & 38 & 35 \\
\hline 2 & 12 & 15 \\
\hline
\end{tabular}

There is no significant difference between the ASA status of patients in both groups.

Table: 6 Weight of patients of A and B

\begin{tabular}{|l|c|c|}
\hline Weight & Group A & Group B \\
\hline Mean & 55.54 & 53.64 \\
\hline SD & 3.21 & 3.56 \\
\hline
\end{tabular}

There is no significant difference between average weight of Group A and Group B

Table-7 Intra-Operative Fentanyl requirement in mcg per hour of surgery

\begin{tabular}{|l|l|l|}
\hline Group & A & B \\
\hline $\begin{array}{l}\text { Fentanyl } \\
\text { requirement. }\end{array}$ & 3.06 & 2.12 \\
\hline SD & 0.62 & 0.46 \\
\hline
\end{tabular}

The intra operative Fentanyl requirement per hour of the patient during surgery was markedly reduced in the group B where Subcostal TAP block was administered as a component of multimodal analgesia. ( $p$ value $<0.02$ ) 
Table 8. Minimum Concentration of Sevoflurane

\begin{tabular}{|l|l|l|}
\hline Group & A & B \\
\hline $\begin{array}{l}\text { Mininum Sevoflurane } \\
\text { Concentration in \%. }\end{array}$ & 1.87 & 1.12 \\
\hline SD & 0.13 & 0.09 \\
\hline
\end{tabular}

Fig:4

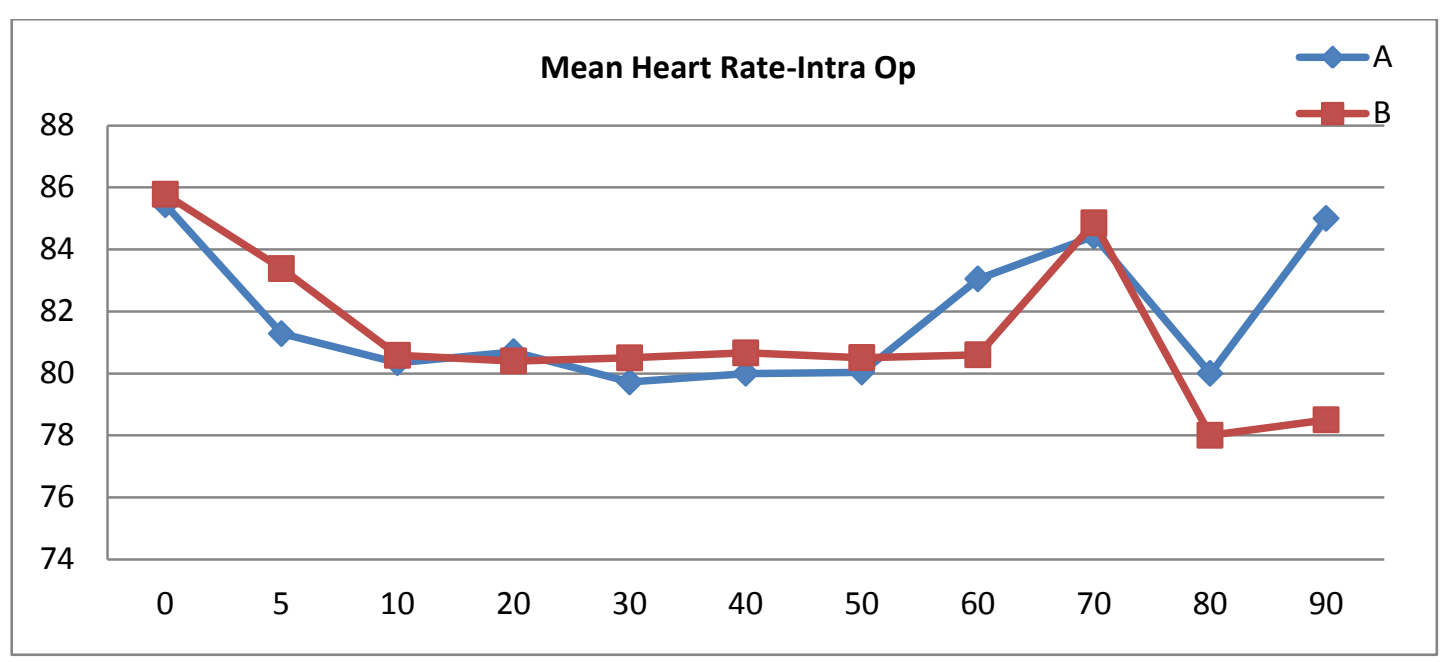

Fig.5

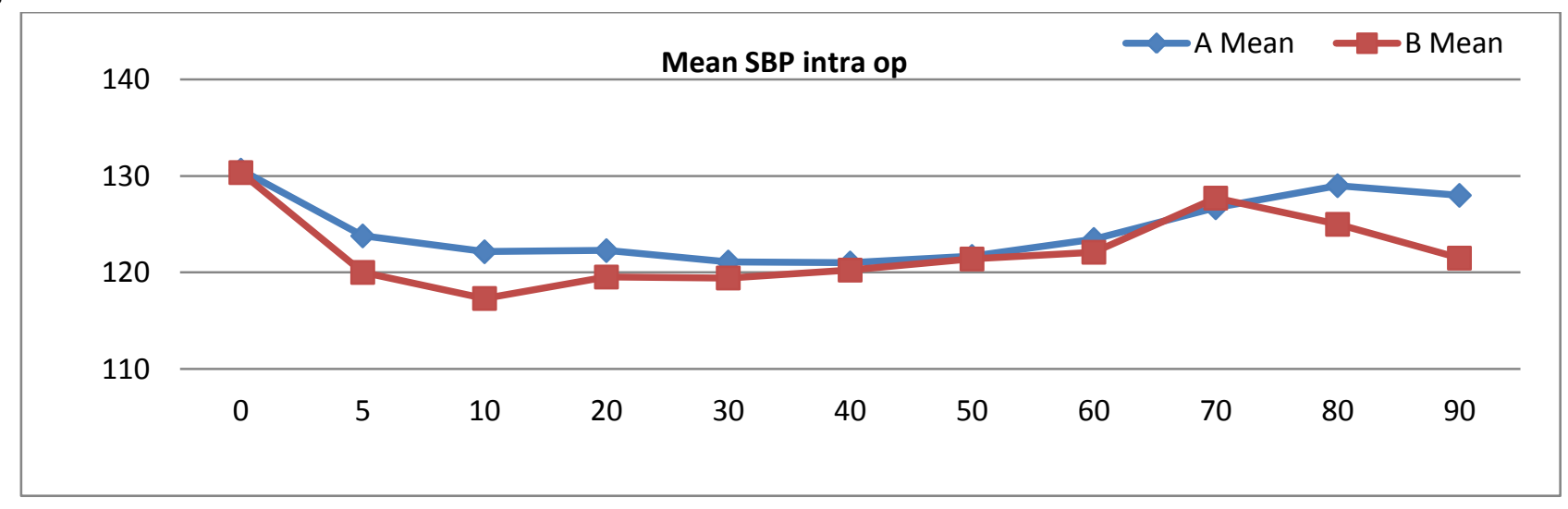

The Minimum concentration of Sevoflurane required to maintain adequate anaesthesia with the standardised anaesthetic protocol was found to be markedly reduced in the TAP Block group, B. ( $p$ value $<0.05$ )

The Intra operative Systolic BP and Heart Rate charts show adequate control of the anaesthetic levels throughout the surgeries in the both Groups of patients. This is established with varying amounts of Fentanyl and Sevoflurane concentrations in different patients.
Table-9 Requirement of Inj. Tramadol IV in $\mathrm{mg} / \mathrm{kg}$ for the first 8 post-operatve hours.

\begin{tabular}{|l|l|l|}
\hline Group & A & B \\
\hline Tramadol in 8 hours $\mathrm{mg} / \mathrm{kg}$ & 1.72 & 0.81 \\
\hline S D & 0.42 & 0.22 \\
\hline
\end{tabular}

There is a statistically significant reduction in the demand of post operative narcotic analgesia in the Group B. (p value < 0.04). Inj. Tramadol $0.5 \mathrm{mg} / \mathrm{kg}$ was administered to both group of patients, to provide a narcotic support when the effect of Fentanyl and Sevoflurane is suddenly withdrawn after recovery from anaesthesia. Many patients in the Group A complained of pain from the port sites 
especially the sensitive umbilical port. This may be due to the inadequate local anaesthetic infiltration done by the surgical team.

\section{DISCUSSION}

Regional analgesia is an important component of multimodal analgesia. During General Anaesthesia, the intra operative analgesia is generally met by the Inhalational agents and the narcotic analgesics. Non-Steroidal Anti Inflammatory Drugs are seldom used for this purpose. Local Anaesthetic infiltration along the incision lines is an accepted technique in many hospitals. But it does not provide analgesia to the full thickness of the abdominal wall like the Transversus Abdominis Plane block.

In the study group B, we have administered Bilateral Subcostal TAP Block 30 minutes before the start of anaesthesia.

This group required statistically significant lower dose of intra operative Fentanyl requirement per hour. ( $p$ value $<0.02$ )

Minimum concentration of Sevoflurane required for the maintenance of anaesthesia, was also statistically lower in the study group B than the control group A. ( $p$ value $<0.05$ )

The port sites were infiltrated with local anaesthetics before closure in both groups. Still there is a marked reduction in the post-operative analgesic requirement for the first 8 hours in the study Group B ( $\mathrm{p}$ value < 0.04 ). This may be due to the pre emptive regional anaesthesia provided by the Bilateral Sub-costal TAP Block administered 30 minutes before the induction of General Anaesthesia. TAP block provides analgesia to the full thickness of the abdominal wall. The port site local anaesthetic infiltration done by the surgical team, on many occasions, were inadequate and to the skin only and left out the full thickness of the abdominal wall.

The study was done in ASA 1 and 2 patients, for ethical reasons. But the reduced requirement of the Inhalational agents is more beneficial to the ASA 3 and 4 patients with poor organ function. Higher concentration of the inhalational agents may lead to the decompensation of these patients. Cardiac compromised patients with low cardiac output states may develop cardiac failure. Healthy young adults can easily tolerate a higher concentration of these agents. So the real benefit of a complimenting subcostal TAP block is for the patients with poor cardiac reserve.

On surgical exploration, a minimally invasive laparoscopic procedure is sometimes found not feasible. Then the surgery is converted to an open procedure. The pain generated during a muscle and nerve cutting subcostal incision is severe and demands a higher concentration of inhalational agents and narcotic analgesics. This increased demand can easily be avoided if a subcostal TAP is done before the procedure. In our study, 8 patients were excluded from the study when the laparoscopic procedure was converted to open; but their anaesthetic demand remained low throughout the surgery.

\section{CONCLUSION}

Complimenting General Anaesthesia with Bilateral Sub-costal Transversus Abdominis Plane block as a mode of multimodal analgesia significantly reduces the intra-operative anaesthetic requirement for Laparoscopic Cholecystectomy. The reduction in the requirement of both the Opioids and the Inhalational agents are statistically significant. This may not make a significant difference in the outcome in an ASA 1 or 2 patient. But in ASA 3and 4 patients with compromised organ function, reduced anaesthetic demand makes a big difference. In those with reduced cardiac reserve, a reduction in the Inhalational anaesthetic concentration is beneficial.

\section{REFERENCES}

1. Hebbard P. Subcostal transversus abdominal plane block under ultrasound guidance. Anesth Analg. 2008;106:67475. [PubMed: 18227342]

2. Barrington MJ, Ivanusic JJ, Rozen WM, Hebbard P. Spread of injectate after ultrasound-guided transversus abdominis 
plane block: a cadaveric study. Anaesthesia. 2009; 64:745-50. [PubMed: 19624629]

3. Abdallah $\mathrm{FW}^{1}$, Chan VW, Brull

R.Transversus abdominis plane block: a systematic review. Reg Anesth Pain Med. 2012 Mar-Apr;37(2):193-209. Doi 10.1097/AAP.0b013e3182429531.

4. Niraj G, Searle A, Mathews M, Misra V, Baban M, Kiani S, et al. Analgesic efficacy of ultrasound-guided transversus abdominis plane block in patients undergoing open appendicectomy. Br J Anaesth. 2009; 103:601-5. [PubMed: 19561014]

5. Mc Donnell JG, O'Donnell B, Curley G, Heffernan A, Power C, Laffey JG. The analgesic efficacy of transversus abdominis plane block after abdominal surgery: A prospective randomized controlled trial. Anesth Analg. 2007;104:1937. [PubMed: 17179269]

6. Mc Donnell JG, Curley G, O’Donnell B, Heffernan A, Power C, Laffey JG. The analgesic efficacy of transversus abdominis plane block after caesarean section: A prospective randomized controlled trial. Anesth Analg. 2008;106:18691. [PubMed: 18165577]

7. El-Dawlatly AA, Turkistani A, Kettner SC, Machata AM, Delvi MB, Thallaj A, et al. Ultrasound guided transversus abdominis plane block: description of a new technique and comparison with conventional systemic analgesia during laparoscopic cholecystectomy. Br J Anaesth. 2009;102:7637. [PubMed: 19376789]

8. S Tolchard, $\mathrm{R}$ Davies, and $\mathrm{S}$ Martindale. Efficacy of the subcostal transversus abdominis plane block in laparoscopic cholecystectomy: Comparison with conventionnal port-site infiltration $\mathrm{J}$ Anaesthesiol Clin Pharmacol. 2012 Jul-Sep; 28(3): 339-343

9. Boddy AP, Metha S, Rhodes M. The effect of intraperitoneal local anaesthesia in laparoscopic cholecystectomy: A systematic review. Anesth Analg. 2006;103:62-8.

10. Erol DD, Yilmaz S, Polat C, Arikan Y. The efficacy of thoracic epidural analgesia in laparoscopic cholecystectomy. Adv Therap. 2008;25:4552. [PubMed: 18227981]

11. Shin $\mathrm{HJ}^{1}$, Oh AY, Baik JS, Kim JH, Han SH, Hwang JW.Ultrasound-guided oblique subcostal transversus abdominis plane block for analgesia after laparoscopic cholecystectomy: a randomized, controlled, observerblinded study. Minerva Anestesiol. 2014 Feb;80(2):185-93. Epub 2013 Oct 31

12. Wu $Y^{1}$, Liu F, Tang $H$, Wang $Q$, Chen $\mathrm{L}, \mathrm{Wu} \mathrm{H}$, Zhang $\mathrm{X}$, Miao J, Zhu M, Hu C, Goldsworthy M, You J, Xu X The analgesic efficacy of subcostal transversus abdominis plane block compared with thoracic epidural analgesia and intravenous opioid analgesia after radical gastrectomy. Anesth Analg. 2013 Aug;117(2):507-13. doi: 10.1213/ANE.0b013e318297fcee. Epub 2013 Jun 6.

13. Prof. H.L. Kaul1 Dr. Neerja Bharti, Monitoring depth of Anaesthesia; Indian J. Anaesth. 200246 (4) : 323-332 323: 